\title{
UV laser radiation inhibits domain inversion in lithium niobate
}

\author{
S. Mailis, A. C. Muir, Y. J. Ying, C. L. Sones, R. W. Eason \\ Optoelectronics Research Centre, University of Southampton \\ Highfield, Southampton, SO17 1BJ, U.K. \\ T. Jungk, A. Hoffmann, E. Soergel \\ Institute of Physics, University of Bonn, Wegelerstrasse 8, 53115 Bonn, Germany \\ S. Mailise-mail address: sm@orc.soton.ac.uk
}

\begin{abstract}
Abtract: Continuous wave UV laser $(\lambda=244 \mathrm{~nm})$ irradiation of the $+\mathrm{z}$ face of lithium niobate single crystals inhibits ferroelectric domain inversion in the volume of the crystal which lies immediately below the UV exposed surface. (C)2008 Optical Society of America OCIS codes: 160.3730 Lithium niobate, 350.3850 Materials processing
\end{abstract}

\section{Introduction.}

Ferroelectric domain engineering in lithium niobate (LN) is a subject of extensive research mainly for the fabrication of quasi-phase-matched (QPM) nonlinear optical devices, for the performance improvement of linear devices and for microstructuring.

Laser illumination of LN crystals has been observed to assist the electric field ferroelectric domain inversion process by reducing locally the value of the coercive field $\left(E_{c}\right)$. Reduction of $E_{c}$ up to two orders of magnitude has been observed in Light Assisted Poling (LAP) experiments [1,2]. The laser radiation which has been used in LAP is normally not significantly absorbed by the crystal $(\lambda=350 \mathrm{~nm}-800 \mathrm{~nm})$ and the inverted ferroelectric domains typically extend throughout the thickness of the crystal.

Here we present UV laser induced inhibition of ferroelectric domain inversion where spatially selective preexposure of the $+\mathrm{z}$ face of a LN crystal inhibits domain inversion in the UV exposed area during electric field poling at a later stage. In these experiments the two steps of i) UV illumination and ii) E-field application are separated; the application of the external electric field can take place long (days) after the UV illumination.

\section{Experimental procedure}

The experimental arrangement for the UV exposure consisted of a set of computer controlled $\mathrm{x}-\mathrm{y}-\mathrm{z}$ translation stages on which $500 \mu \mathrm{m}$ thick crystal samples (source; Crystal Technology Inc, USA) are mounted and scanned in front of the focussed UV laser beam. The laser source was a frequency doubled Ar: ion laser at $\lambda=244 \mathrm{~nm}$ which was focussed down to a spot size of $\sim 2.5 \mu \mathrm{m}$. A computer controlled mechanical shutter was also used to control the exposure time. In the experiments presented here two types of exposures were performed always on the $+z$ face of the samples: i) arrays of linear tracks where the crystal is translated linearly at a constant speed and the length of these tracks varied between $0.5 \mathrm{~mm}$ to $5 \mathrm{~mm}$ and ii) arrays of exposures where the crystal was static during the exposure, the duration of which was controlled by the mechanical shutter.
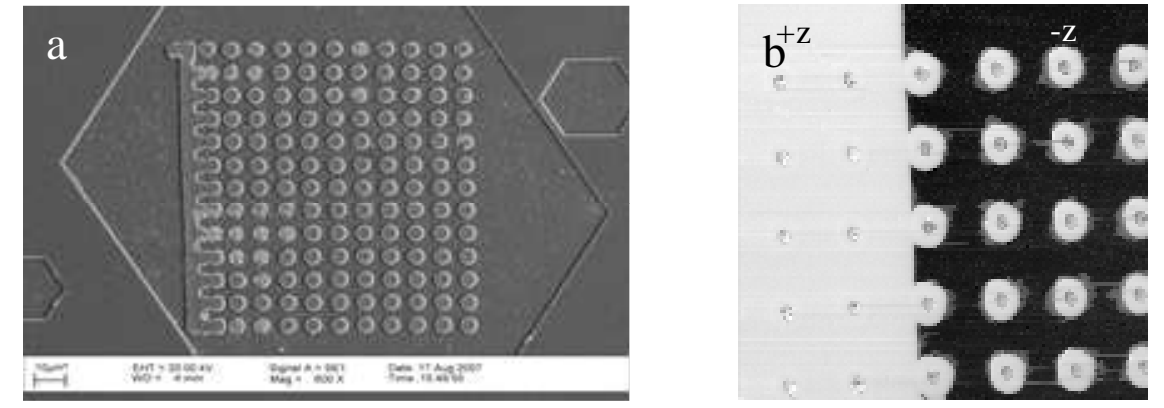

Figure1. a) SEM image of the HF-etched $+z$ face of a UV exposed and poled congruent LN sample. The array of dots correspond to the UV exposed areas. b) Piezoresponse map of the $+\mathrm{z}$ face of a UV dot-exposed and partially poled LN sample. The black and white areas correspond to $-\mathrm{z}$ and $+\mathrm{z}$ faces respectively. The PFM scan size is $58 \mu \mathrm{m} \times 58 \mu \mathrm{m}$ 
After UV illumination the crystal sample was domain-inverted by applying a uniform external electric field exceeding the $\mathrm{E}_{\mathrm{c}}$ value which for congruent $\mathrm{LN}$ crystals is $\sim 22 \mathrm{kV} / \mathrm{mm}$. The inverted domain topography of the UV exposed face was investigated by scanning electron microscopy (SEM) after brief etching ( 15 minutes) in pure HF acid. Figure 1a shows an array of upstanding dots which corresponds to static exposures and seems to have maintained their original domain orientation. The area surrounding these features is domain inverted and now corresponds to $\mathrm{a}-\mathrm{z}$ face. This is shown clearly in figure 1a where the dot array appears to be surrounded by the newly poled hexagonally shaped domain.

Piezoresponse force microscopy (PFM) was used to verify the ferroelectric domain polarity of the features which were observed in the SEM images. The PFM scan which is presented in figure 1b shows the domain mapping of a spot illuminated surface that has been partially domain inverted. In the PFM image the white area corresponds to $\mathrm{a}+\mathrm{z}$ face and the black area corresponds to $\mathrm{a}-\mathrm{z}$ face as indicated in the figure. The right part of the image has been domain inverted and appears black. The white dots observed within the black background corresponds to UV illuminated spots which have resisted poling and show the same contrast as the left part of the image which has also not been domain inverted. The vertical contrast divide corresponds to the domain wall between the newly poled domain on the right hand side and the original domain on the left. The dot-exposed array extends on both sides of the domain wall. Interestingly the area in the centre of the exposed dots appears grey indicating the presence of an amorphous layer as a result of surface melting in the more intense central area of the Gaussian intensity profile of the laser spot.

\section{Discussion}

The results presented here show that the effective coercive field increases in the UV exposed areas which is opposite to what has been observed in all LAP experiments. The difference in this case is that short wavelength UV radiation is used which is highly absorbed by the crystal. UV radiation at $\lambda=244 \mathrm{~nm}$ is absorbed in the first few tens of nanometers [3] in LN and it is capable of producing photo-excited charge and heat. Our hypothesis is that the photoexcited charges diffuse through the affected volume and get trapped in crystal defects thus creating suitable electrostatic termination points for the propagating bulk domain which has nucleated on the original $-\mathrm{z}$ face (during the poling process) and propagates towards the original $+\mathrm{z}$ face. As a result of this process the surface and the volume of the crystal below the UV exposed areas maintain the original domain polarity.

The depth of this non-inverted part is currently unknown. However, observation of the PFM scan in figure 1b shows that the non-inverted domains on the right hand side have the same contrast as the bulk non-inverted domain on the left hand side of the image. The depth sensitivity of the PFM is of order $\sim 100 \mathrm{~nm}$ which is roughly 3 times the radius of curvature of the AFM tip which is used [4]. Any domain deeper than $\sim 100 \mathrm{~nm}$ would appear as bulk hence this corresponds to a minimum depth for the domain inverted features which we observe.

\section{Conclusions}

The exposure of the $+\mathrm{z}$ surface of congruent lithium niobate single crystals to UV laser radiation $(\lambda=244 \mathrm{~nm})$ is shown here to locally inhibit domain inversion during the process of E-field poling. The effect was investigated by SEM of HF etched samples which showed differentially etched features corresponding to the UV irradiated areas. The domain inhibition was further verified by piezoresponse microscopy. PFM also provides a minimum depth of these non-inverted domains of $\sim 100 \mathrm{~nm}$ as deduced from the depth sensitivity of the instrument. UV induced poling inhibition is attributed to the redistribution of diffused photo-induced charges and subsequent trapping in crystal defect sites which provide electrostatic termination points for propagating bulk domains during the E-field poling process.

Depending on the depth of these domain structures they can be used in nonlinear waveguide devices (waveguide PPLNs) however, this effect when combined with chemical etching provides a very versatile tool for the fabrication of surface micro/nano-structuring of excellent optical quality in lithium niobate.

\section{References}

[1] C.L.Sones, M.C.Wengler, C.E.Valdivia, S.Mailis, R.W.Eason, K.Buse, "Light-induced order-of-magnitude decrease in the electric field for domain nucleation in MgO-doped lithium niobate crystals" Appl. Phys. Lett. 86, pp.212 (2005)

[2] C.E.Valdivia, C.L.Sones, S.Mailis, J.D.Mills, R.W.Eason "Ultrashort-pulse optically-assisted domain engineering in lithium niobate" Ferroelectrics 340, pp.75-82 2006

[3] A. M. Mamedov, "Optical properties (VUV region) of LiNbO3," Optics and Spectroscopy 56, pp 645 - 649 (1984)

[4] T. Jungk, A. Hoffmann, and E. Soergel "Impact of the tip radius on the lateral resolution in piezoresponse force microscopy" arXiv:condmat/0703793v1 